\title{
Pectolinarigenin promotes functional recovery and inhibits apoptosis in rats following spinal cord injuries
}

\author{
BIN WU and JIE LIANG \\ Department of Orthopedic Surgery, The People's Hospital of China Three Gorges University, \\ Yichang, Hubei 443000, P.R. China
}

Received May 31, 2018; Accepted November 9, 2018

DOI: $10.3892 /$ etm.2019.7456

\begin{abstract}
Spinal cord injury (SCI) is a devastating neurological injury that frequently leads to neurological defects and disabilities. The only effective pharmacotherapy currently available is methylprednisolone (MP), which is controversial due to its high incidence of complications, adverse events and ultimately limited efficacy in SCI. Therefore, the development of alternative therapeutic agents for the treatment of SCI is of great clinical significance. In the present study, an acute SCI rat model was induced and, following a modified Allen method, the function of pectolinarigenin (PG) in SCI was investigated. A total of 36 rats were randomly divided into 6 groups ( $n=6$ in each group); a sham surgery group and an SCI + saline group were used as negative controls and an $\mathrm{SCI}+\mathrm{MP}$ (30 mg/kg) group was used as a positive control. The remaining animals were subdivided into three groups: $\mathrm{SCI}+\mathrm{PG}(10 \mathrm{mg} / \mathrm{kg}) ; \mathrm{SCI}+\mathrm{PG}(30 \mathrm{mg} / \mathrm{kg}) ;$ and $\mathrm{SCI}+\mathrm{PG}$ $(50 \mathrm{mg} / \mathrm{kg})$. Basso-Beattie-Bresnahan locomotor rating scoring was performed to assess functional recovery. Nissl staining and TUNEL staining were used to evaluated neuronal lesion volume and apoptosis, respectively. The results demonstrated that PG significantly improved functional recovery and reduced tissue loss, and neuronal apoptosis. Furthermore, a western blotting assay was conducted to measure the expression of genes associated with apoptosis. The data suggested that PG downregulated the activated caspase-3, caspase- 9 and poly-ADP-ribose polymerase expression and reduced the Bax: $\mathrm{Bcl} 2$ ratio. The findings of the present study suggested that $\mathrm{PG}$ may exert a protective effect against SCI in rats, potentially by inhibiting neuronal apoptosis and PG may therefore serve as a novel therapeutic agent against SCI.
\end{abstract}

Correspondence to: Dr Jie Liang, Department of Orthopedic Surgery, The People's Hospital of China Three Gorges University, 2 Jiefang Road, Yichang, Hubei 443000, P.R. China

E-mail: jieliang33@hotmail.com

Key words: spinal cord injury, apoptosis, pectolinarigenin

\section{Introduction}

Spinal cord injury (SCI) is a traumatic event resulting in serious disability and neurological deficits, which results in a high economic burden and huge psychological pressures for individuals and their families (1). The greatest harm following SCI is nerve damage $(2,3)$. Previous studies have demonstrated that nerve injury is caused by two mechanisms, comprising primary mechanical injury and secondary injury $(4,5)$. Secondary injury is triggered by a series of cellular events and gene expression alterations, accompanied by a variety of extended neuropathophysiological alterations and inflammatory responses over a few minutes to a few weeks following the initial injury $(6,7)$. Apoptosis is the process of programmed cell death and serves a crucial role in secondary lesions (8). Caspases have previously been observed to exhibit proteolytic activity and are able to cleave substrate proteins at aspartic acid residues, and serve an essential role in apoptosis (9). Caspase-3 is activated by initiator caspases, including caspase-8, caspase- 9 and caspase-10, and is well-established as the most critical member of the executioner caspases; it can induce cell apoptosis irreversibly by cleaving substrate proteins including poly-adenosine diphosphate-ribose polymerase (PARP) (10). The anti-apoptotic protein $\mathrm{Bcl}-2$ and pro-apoptotic protein Bax belong to the Bcl-2 family, and serve a vital role in cell apoptosis by regulating the permeability of the mitochondrial membrane (11).

SCI is a worldwide medical problem, there are 179,312 novel traumatic spinal cord injury cases per year (12). No effective pharmacotherapy is currently available other than methylprednisolone (MP), which is a potent anti-inflammatory agent (13). However, the use of MP is subject to much debate, due to its limited efficacy and numerous adverse side effects (14). At present, there is still no effective, standardized method of treating SCI, therefore the identification and development of novel therapeutics would be of great clinical significance in the field of SCI treatment. Pectolinarigenin (PG) is an active component of medicinal thistle plants and Eupatorium odoratum L., and exerts significant anti-inflammatory and hemostatic effects (15). However, there are few studies examining the function of PG (16). Therefore, the aim of the present study was to investigate the role of PG in SCI.

The data obtained in the present study suggested that PG significantly improved functional recovery and reduced 
tissue loss, and neuronal apoptosis in rats with SCI. In addition, the results of western blotting assay demonstrated that the expression of pro-apoptotic proteins (caspase-3, caspase-9 and PARP) were downregulated and the $\mathrm{Bax}: \mathrm{Bcl} 2$ ratio was reduced following treatment with $\mathrm{PG}$.

\section{Materials and methods}

Animals and grouping. A total of 36 adult male Sprague-Dawley rats, obtained from the Shanghai SLAC Laboratory Animal Co., Ltd., (Shanghai, China), weighing between 200 and $250 \mathrm{~g}$ were used for the experiments. All rats were housed at $22-25^{\circ} \mathrm{C}$, at $40-60 \%$ humidity with a 12 -h light-dark cycle and free access of food and water. For the spinal cord injury (SCI) rat model, a dorsal longitudinal incision was made to expose T9-T11 following the administration of anesthetics. A laminectomy was subsequently performed at T10 and the modified Allen method was conducted to induce acute SCI as described previously, dropping a $10 \mathrm{~g}$ rod from a $4 \mathrm{~cm}$ height onto the dorsal surface of the spinal cord $(17,18)$. Rats in the sham-surgery group received a laminectomy only. All animal handling procedures and experimental protocols were consistent with the National Institutes of Health Guide for the Care and Use of Laboratory Animals (GB 14925-2001).

The animals were randomly divided into six experimental groups ( $n=6$ in each group) as follows: i) Sham-surgery group (in which rats were only treated with a laminectomy without SCI for comparison); ii) SCI + saline; iii) SCI + low-dose PG $(10 \mathrm{mg} / \mathrm{kg})$; iv) SCI + middle-dose PG $(30 \mathrm{mg} / \mathrm{kg})$; v) $\mathrm{SCI}+$ high-dose $\mathrm{PG}(50 \mathrm{mg} / \mathrm{kg})$ and vi) $\mathrm{SCI}+\mathrm{MP}(30 \mathrm{mg} / \mathrm{kg})$, which was used as a positive control. PG or MP was administered to rats intraperitoneally, every 2 days.

Behavioral assessment. Basso-Beattie-Bresnahan (BBB) locomotor rating scoring was performed to assess the functional recovery of the rat hind limbs. The neurological outcome for each animal was examined in an open field using the BBB locomotor rating scale, ranging from 0 (complete paralysis) to 21 (normal locomotion) (19). Scores from 0 to 7 were considered to indicate the early stages of recovery and were used to judge the joint activities of the hind limbs; scores from 8 to 13 indicated the intermediate recovery phase, and were used to assess the gait of the animals and the coordination of the hind limbs. Finally, scores from 14 to 21 were considered to indicate the late phase of recovery and were used to assess the fine motions of the paw during animal movement. BBB testing was performed on days $1,3,7,14,21$ and 28 following spinal cord surgery. Each rat was scored once a day in double-blind model for a period of 4 min by two examiners and an average value per animal was calculated.

Nissl staining. Following the behavioral tests, tissue blocks comprising $1-\mathrm{cm}$ sections of spinal cord centered on the lesion site were embedded in paraffin. Sample sections $(5 \mu \mathrm{m})$ of the lesion site were stained for Nissl with cresyl-violet acetate (Sigma-Aldrich, Merck KGaA, Darmstadt, Germany) at room temperature for $10 \mathrm{~min}$. The tissue sections were cleared using xylene and rehydrated with anhydrous ethanol at 95,80 , and $70 \%$ alcohol and double-distilled water. Sections were then stained in $0.1 \%$ cresyl-violet acetate (Sigma-Aldrich; Merck $\mathrm{KGaA}$ ) at room temperature for $10 \mathrm{~min}$. The tissues were rinsed in double-distilled water and differentiated in $70 \%$ ethanol with acetic acid for $2 \mathrm{~min}$. The tissues were subsequently dehydrated with 70, 80 and 95\% alcohol, washed in xylene, and placed on a cover-slip with slide mounting medium. Image-Pro Plus 6.0 (Media Cybernetics, Inc., Rockville, MA, USA) software was used to count the number of surviving neurons.

Terminal dUTP nick-end labeling (TUNEL) staining. The TUNEL assay was performed to assess cell apoptosis. Spinal cords were excised and fixed in $4 \%$ paraformaldehyde overnight at room temperature and embedded in paraffin. Samples were stained with terminal TUNEL kit(40307ES20; Yeasen, Shanghai, China) according to the manufacturer's protocol. Sample sections $(5 \mu \mathrm{m})$ were cleared using xylene and rehydrated with anhydrous ethanol at 90, 80 and 70\% alcohol and deionized water. Sections were then stained with an Alexa Fluor 488-12-dUTP Labeling Mix (Yeasen) at $37^{\circ} \mathrm{C}$ for $1 \mathrm{~h}$. TUNEL-stained slides were observed under fluorescence microscopy (Olympus Corporation, Tokyo, Japan) at x400 magnification, and five fields of view for each section were randomly selected for subsequent calculations. The number of TUNEL positive cells was counted, and the average value was collected. Data were collected from three independent experimental repeats.

Western blot analysis. Total protein was extracted from T9-T11 spinal cord tissues using a lysis buffer, which contained $50 \mathrm{mM}$ Tris- $\mathrm{HCl}$, pH 7.4; 5 mM EDTA; $1 \%$ (v/v) NP-40 with $1 \mathrm{mM}$ phenylmethylsulfonyl fluoride. A bicinchoninic assay kit (Bio-Rad Laboratories, Inc., Hercules, CA, USA) was used to measure the protein concentration. Protein samples (40 $\mu \mathrm{g} /$ lane) were separated via SDS-PAGE (10\% resolving gels, $4 \%$ stacking gels) and blotted onto polyvinylidene difluoride membranes (Thermo Fisher Scientific, Inc., Waltham, MA, USA). The membranes were blocked in 5\% non-fat milk for $1 \mathrm{~h}$ at room temperature, followed by incubation overnight at $4^{\circ} \mathrm{C}$ with the primary antibodies. Subsequently, secondary antibodies were incubated at room temperature for an additional $2 \mathrm{~h}$. Chemiluminescent signals were visualized using the ECL detection reagent (EMD Millipore, Billerica, MA, USA). Image J software 1.4 (National Institutes of Health, Bethesda, MD, USA) was used to analyze the relative protein expression. The antibodies used are listed as follow: Cleaved-caspase-3 (1:500; cat. no. 9664S; Cell Signaling Technology, Inc., Danvers, MA, USA), cleaved-caspase-9 (1:500; cat. no. 9509), cleaved-PARP (1:500; cat. no. 94885), Bcl-2 (1:1,000; cat. no. 3498), Bax (1:1,000; cat. no. 14796; all Cell Signaling Technology, Inc.), $\beta$-actin (1:1,000; cat. no. sc-517582), horseradish peroxidase (HRP) conjugated rabbit anti-mouse immunoglobulin G (IgG; 1:5,000; cat. no. sc-358914), HRP-mouse anti-rabbit IgG (1:10,000; cat. no. sc-2357; all Santa Cruz Biotechnology, Inc.).

Statistical analysis. Data are presented as the mean \pm standard deviation and each experiment was repeated three times. GraphPad Prism version 6 (GraphPad Software Inc., La Jolla, CA, USA) was used to perform the statistical analyses. One-way analysis of variance was used to compare the significant differences among multiple groups, followed by Tukey's post-hoc analysis. $\mathrm{P}<0.05$ was considered to indicate a statistically significant difference. 


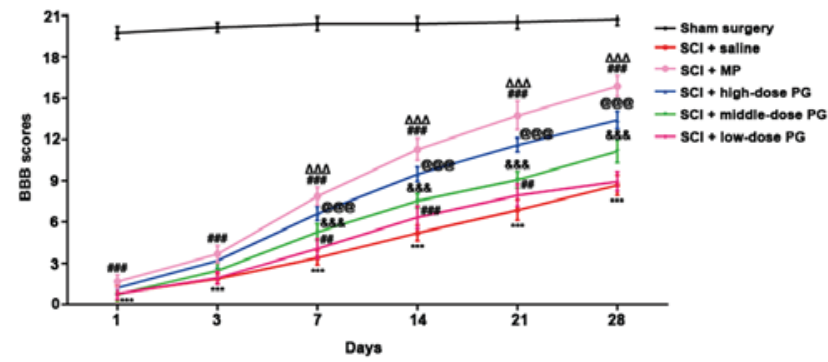

Figure 1. PG promotes functional recovery in rats following SCI. BBB locomotor rating scoring was performed on days 1, 3, 7, 14, 21 and 28 following SCI. ${ }^{* * * *} \mathrm{P}<0.001 \mathrm{SCI}+$ saline vs. Sham surgery, ${ }^{\Delta \Delta \Delta \mathrm{P}<0.001}$ $\mathrm{SCI}+\mathrm{MP}$ vs. high-dose PG $(50 \mathrm{~kg} / \mathrm{ml})$, ${ }^{@ @ @ ~} \mathrm{P}<0.001 \mathrm{SCI}+$ high-dose PG $(50 \mathrm{~kg} / \mathrm{ml})$ vs. SCI + saline, \&\&\& $<0.001 \mathrm{SCI}+$ middle-dose PG vs. SCI + low-dose PG, ${ }^{\# \#} \mathrm{P}<0.01,{ }^{\# \# \#} \mathrm{P}<0.001 \mathrm{SCI}+$ low-dose $\mathrm{PG}$ or $\mathrm{SCI}+\mathrm{MP}$ vs. SCI + saline. BBB, Basso-Beattie-Bresnahan; SCI, spinal cord injury; MP, methylprednisolone; $\mathrm{PG}$, pectolinarigenin.

\section{Results}

Protective effects of $P G$ on functional recovery in rats following acute SCI. In order to assess the effects of PG on functional recovery following acute $\mathrm{SCI}$ in each group, BBB locomotor rating scoring was performed on days 1, 3, 7, 14, 21 and 28 following SCI (Fig. 1). The BBB locomotor test is a sensitive and reliable method of detecting locomotor function following SCI. MP and saline were used as a positive control and normal control, respectively. Different doses of PG $(10,30$ and $50 \mathrm{mg} / \mathrm{kg}$ ) were administered to the appropriate groups, and the mean BBB scores were collected. It was observed that the middle-dose PG group and high-dose PG group exhibited functional recovery by days 7 and 3 respectively, and the effect lasted until day 28 following SCI (Fig. 1). However, BBB scores in the low-dose PG group were significantly increased only on days 7, 14 and 21 ( $\mathrm{P}<0.01$; Fig. 1). From day 7-28, the scores in the high-dose PG group were increased compared with the middle-dose PG group and the differences were statistically significant $(\mathrm{P}<0.001$; Fig. 1). Similarly the middle-dose $\mathrm{PG}$ group exhibited higher scores than low-dose PG group (Fig. 1). These data demonstrated that $\mathrm{PG}$ exerted protective effects on the spinal cord in a dose-dependent manner. Treatment with PG $50 \mathrm{mg} / \mathrm{kg}$ was demonstrated to be the most effective dose, with an effect comparable to that of MP at $30 \mathrm{mg} / \mathrm{kg}$.

Protective effects of $P G$ on neuron damage in the spinal cord of SCI rats. In order to further examine the underlying mechanism of the protective function of PG in SCI, Nissl staining was performed to observe any potential neuroprotective effects on day 28 post-SCI. Nissl staining indicated that neurons in the sham group displayed an integrative and granular-like morphology. The SCI + saline group exhibited significantly fewer surviving neurons in the lesions of the spinal cord compared with the sham group $(\mathrm{P}<0.001$; Fig. $2 \mathrm{~A})$. In addition, the neurons appeared swollen and exhibited irregular morphologies (Fig. 2A). The number of surviving neurons increased significantly at 28 days post-SCI in the three PG-treated groups and the SCI + MP (30 mg/kg) group $(\mathrm{P}<0.001$; Fig. 2A). Taken together, these data indicated that PG intervention increased the number of surviving neurons, normalized the morphology of neurons and restored neuronal function, which is represented by the increased number of Nissl bodies. In addition, this experiment revealed that PG exhibited a dose-dependent effect, with higher PG resulted in better neuroprotective effect.

$P G$ inhibits cell apoptosis in rats following SCI. TUNEL staining was performed to assess the effect of PG on apoptotic cells following SCI. The results demonstrated that in the SCI + saline group, positive cells were significantly increased and widely distributed, compared with the sham group ( $\mathrm{P}<0.001$; Fig. 2B). The different doses of PG markedly reduced the number of TUNEL-positive neurons when compared with the SCI + saline group (Fig. 2B). The results exhibited a certain level of dose-dependent efficacy; however there was no statistically significant difference between the middle-dose and low-dose PG groups (Fig. 2B). Subsequently, western blot analysis was performed to detect the expression of apoptosis-associated proteins at day 28 post-SCI. The results revealed that the expression of activated caspase-3, caspase-9 and cleaved-PARP were dramatically increased in the SCI + saline group, compared with the Sham surgery group (Fig. 3). Treatment with PG markedly downregulated the levels of activated caspase-3, caspase-9 and cleaved-PARP (Fig. 3). Additionally, the expression of Bcl-2 was decreased, whereas the $\mathrm{Bcl} 2$ level was increased with the increasing PG concentration (Fig. 3). These data demonstrated that PG inhibited cellular apoptosis by downregulating cleaved caspase-3 and -9 and PARP expression and reducing the Bax: $\mathrm{Bcl} 2$ ratio.

\section{Discussion}

In present study, acute spinal cord injury was induced in a rat model and a modified version of the Allen method was conducted, in order to investigate the role of PG in SCI. MP, the most widely used clinical medicine for the treatment of SCI (13), was used as a positive control drug. The BBB locomotor test was performed to detect the motor function following SCI and the results demonstrated that PG exerted protective effects on the spinal cord. Among the different PG treatment groups, $50 \mathrm{mg} / \mathrm{kg}$ was determined to be the most effective dose, with similar effects to those of MP at $30 \mathrm{mg} / \mathrm{kg}$. Nissl staining confirmed that PG markedly increased the number of surviving neurons, normalized the morphology of neurons and restored neuronal function.

Secondary injury may cause nerve damage, accompanied by a variety of cellular events and occurs minutes to weeks following initial mechanical injury, eventually culminating in damage to tissues (6). Due to the irreversibility of the initial injury, the majority of studies have focused on the therapy and mechanisms of secondary injury (20-22). Apoptosis, which is principally mediated by the caspase and $\mathrm{Bcl}-2$ families, serves a crucial role in secondary lesions and culminates in the cell death of neurons $(23,24)$. The loss of neuronal cells that are not replaced following injury may contribute greatly to disability following SCI $(7,25)$. Taking this into account, the present study further investigated the possible underlying mechanism of the protective effects of PG on neuronal cells by performing TUNEL staining to assess cell apoptosis following SCI. The results demonstrated that treatment 


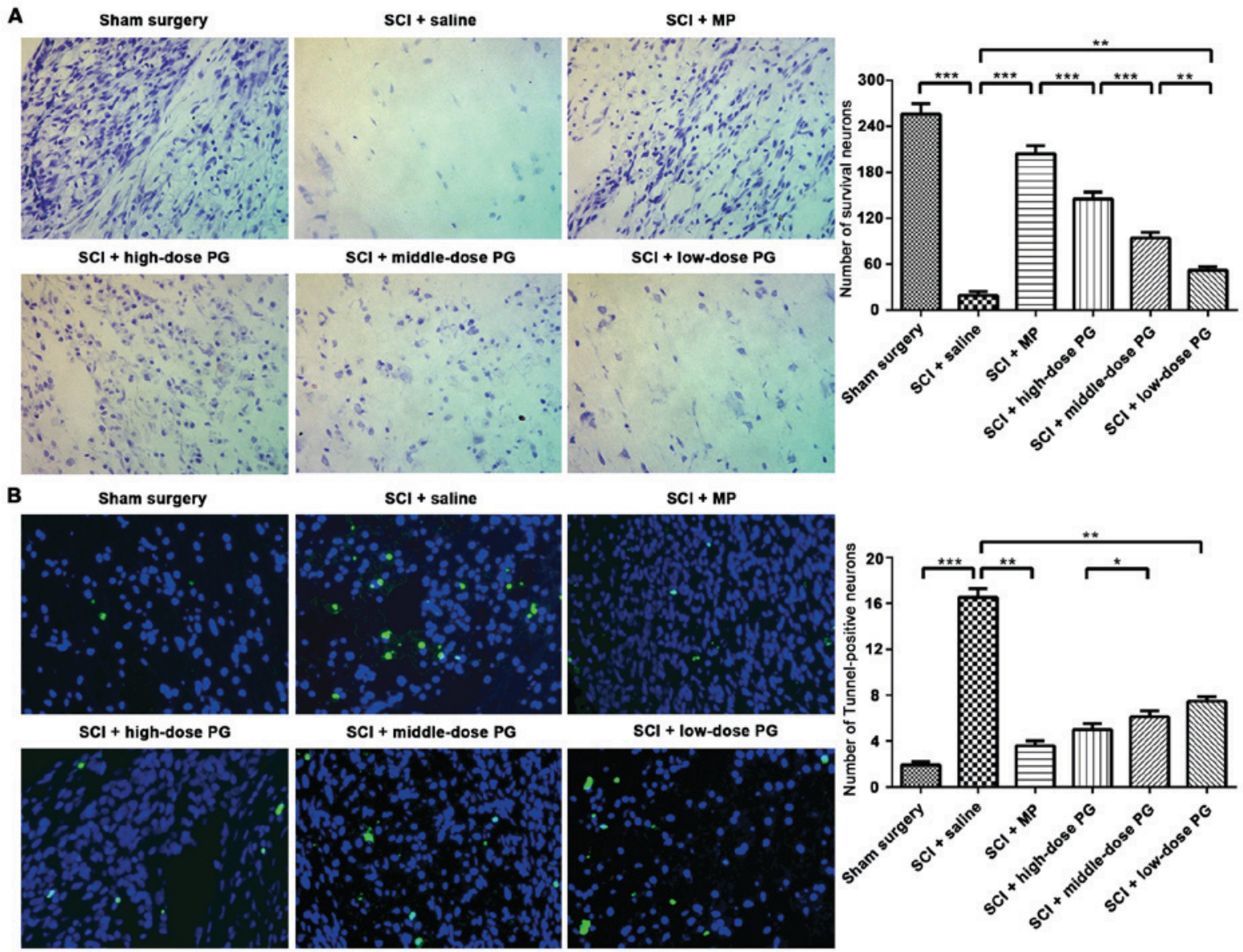

Figure 2. PG prevents neuronal damage in the spinal cords of SCI rats. (A) Nissl staining was performed on day 28 post-SCI. (B) Terminal dUTP nick-end labeling staining was performed on day 28 post SCI. Samples were observed at magnification, $\mathrm{x} 400 .{ }^{*} \mathrm{P}<0.05,{ }^{* *} \mathrm{P}<0.01$ and ${ }^{* * * *} \mathrm{P}<0.001$. SCI, spinal cord injury; MP, methylprednisolone; $\mathrm{PG}$, pectolinarigenin.
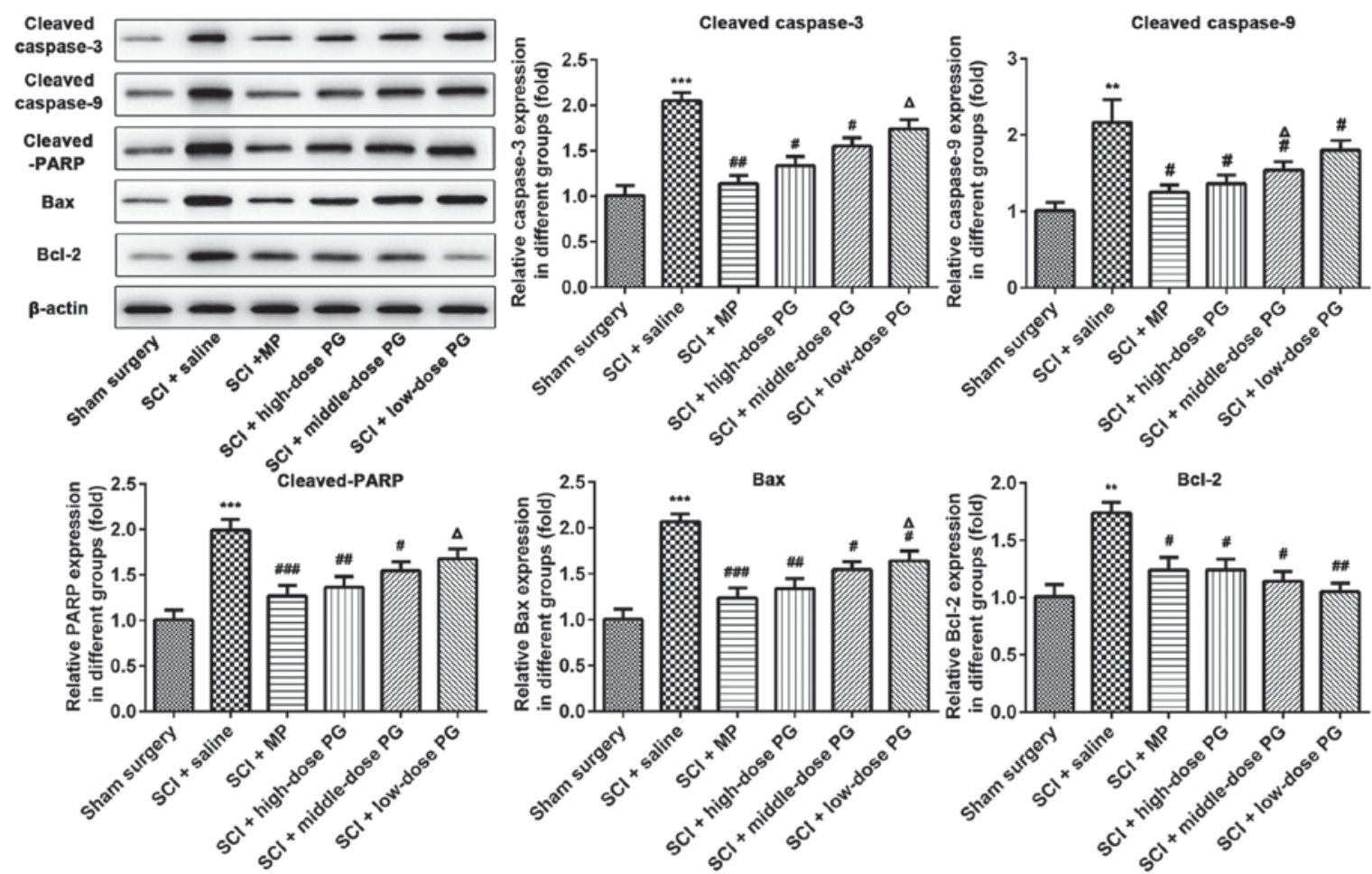

Figure 3. Apoptosis-associated protein and gene expression at day 28 post-SCI. Western blot analysis was performed to detect the protein expression of cleaved caspase-3, caspase-9, cleaved PARP, Bax and Bcl-2. ${ }^{* *} \mathrm{P}<0.01,{ }^{* * *} \mathrm{P}<0.001$ vs. Sham surgery; ${ }^{\# \mathrm{P}}<0.05$, ${ }^{\# \#} \mathrm{P}<0.01$, ${ }^{\# \#} \mathrm{P}<0.001$ vs. SCI + saline; ${ }^{\Delta} \mathrm{P}<0.05$ vs. SCI + MP. SCI, spinal cord injury; MP, methylprednisolone; PG, pectolinarigenin; PARP, poly-ADP-ribose polymerase. 
with PG reduced the number of TUNEL-positive neurons, suggesting that PG inhibited cell apoptosis in rats following SCI. In addition, the expression levels of the pro-apoptotic proteins Bax, cleaved caspase-3 and 9, and cleaved-PARP were identified to be decreased, and the anti-apoptotic protein $\mathrm{Bcl}-2$ was upregulated, following treatment with PG.

Numerous studies have reported the limitations and side effects of MP, which has led to controversy regarding the clinical application of MP for the treatment of SCI; however, at present, there are no suitable effective drugs to replace MP (26-28). The results of the present study suggested that PG may have a protective effect against SCI in rats, potentially by inhibiting neuronal apoptosis. There are few studies on PG and a lot of those that do exist focus on its antitumor activity (29-31). For example, a previous study on nasopharyngeal carcinoma demonstrated that PG activates the caspase signaling pathway and promotes cell apoptosis, further inhibiting tumor growth in nasopharyngeal carcinoma (32). Zhang et al (29) suggested that PG prevents cancer aggressiveness by inhibiting signal transducer and activator of transcription 3 activity in osteosarcoma. By contrast, the data obtained in the present study suggest that PG has a protective effect against SCI in rats, potentially by inhibiting neuronal apoptosis. Notably, Csupor et al (33) identified that PG, extracted from Centaurea sadleriana, exhibited anti-inflammatory activities, which is clinically relevant as inflammation is another critical event in secondary injury following SCI (34). Several drugs have been assessed in experimental and clinical trials that are aimed at reducing the inflammatory response in SCI (35-37). However, whether PG exhibits a role in SCI-induced inflammation requires further study.

Taking the inefficiency and adverse effects of MP into consideration, the identification and development of novel medicines is of great clinical significance in the field of SCI treatment. Although further studies are required, the findings obtained in the current study suggested that PG promotes functional recovery and prevents neuronal damage in rats following SCI by inhibiting apoptosis, at least partially. Therefore PG may serve as a potential therapeutic agent for the treatment of SCI.

\section{Acknowledgements}

Not applicable.

\section{Funding}

No funding was received.

\section{Availability of data and materials}

The datasets used and/or analyzed during the current study are available from the corresponding author on reasonable request.

\section{Authors' contributions}

BW and JL conceived and designed the experiments, and performed data analysis. JL wrote the manuscript and BW reviewed and edited the manuscript.

\section{Ethics approval and consent to participate}

The present study was approved by the institutional Animal Care and Use Committee of the People's Hospital of China Three Gorges University.

\section{Patient consent for publication}

Not applicable.

\section{Competing interests}

The authors declare that they have no competing interests.

\section{References}

1. Calancie B, Molano MR and Broton JG: Epidemiology and demography of acute spinal cord injury in a large urban setting. J Spinal Cord Med 28: 92-96, 2005.

2. Fox IK, Davidge KM, Novak CB, Hoben G, Kahn LC, Juknis N, Ruvinskaya R and Mackinnon SE: Nerve transfers to restore upper extremity function in cervical spinal cord injury: Update and preliminary outcomes. Plast Reconstr Surg 136: 780-792, 2015.

3. Moulin P, Gohritz A and Meunzel J: Spinal cord injury: Still an interdisciplinary challenge [corrected]. Orthopade 43: 625-635, 2014 (In German).

4. Choi DW and Rothman SM: The role of glutamate neurotoxicity in hypoxic-ischemic neuronal death. Annu Rev Neurosci 13: 171-182, 1990.

5. Fehlings MG and Nguyen DH: Immunoglobulin G: A potential treatment to attenuate neuroinflammation following spinal cord injury. J Clin Immunol 30 (Suppl 1): S109-S112, 2010.

6. Song Y, Zeng Z, Jin C, Zhang J, Ding B and Zhang F: Protective effect of ginkgolide $\mathrm{B}$ against acute spinal cord injury in rats and its correlation with the Jak/STAT signaling pathway. Neurochem Res 38: 610-619, 2013.

7. Bareyre FM and Schwab ME: Inflammation, degeneration and regeneration in the injured spinal cord: Insights from DNA microarrays. Trends Neurosci 26: 555-563, 2003.

8. Liu C, Shi Z, Fan L, Zhang C, Wang K and Wang B: Resveratrol improves neuron protection and functional recovery in rat model of spinal cord injury. Brain Res 1374:100-109, 2011.

9. Shalini S, Dorstyn L, Dawar S and Kumar S: Old, new and emerging functions of caspases. Cell Death Differ 22: 526-539, 2015.

10. Slee EA, Adrain C and Martin SJ: Executioner caspase-3, -6, and -7 perform distinct, non-redundant roles during the demolition phase of apoptosis. J Biol Chem 276: 7320-7326, 2001.

11. Cory S and Adams JM: The Bcl2 family: Regulators of the cellular life-or-death switch. Nat Rev Cancer 2: 647-656, 2002.

12. Meijles DN and Pagano PJ: Nox and inflammation in the vascular adventitia. Hypertension 67: 14-19, 2016.

13. Fehlings MG, Wilson JR and Cho N: Methylprednisolone for the treatment of acute spinal cord injury: Counterpoint. Neurosurgery 61 (Suppl 1): S36-S42, 2014.

14. Samano C, Kaur J and Nistri A: A study of methylprednisolone neuroprotection against acute injury to the rat spinal cord in vitro. Neuroscience 315: 136-149, 2016.

15. Lim H, Son KH, Chang HW, Bae K, Kang SS and Kim HP: Anti-inflammatory activity of pectolinarigenin and pectolinarin isolated from Cirsium chanroenicum. Biol Pharm Bull 31: 2063-2067, 2008.

16. Lee S, Lee DH, Kim JC, Um BH, Sung SH, Jeong LS, Kim YK and Kim SN: Pectolinarigenin, an aglycone of pectolinarin, has more potent inhibitory activities on melanogenesis than pectolinarin. Biochem Biophys Res Commun 493: 765-772, 2017.

17. Perot PL Jr, Lee WA, Hsu CY, Hogan EL, Cox RD and Gross AJ: Therapeutic model for experimental spinal cord injury in the rat: I. Mortality and motor deficit. Cent Nerv Syst Trauma 4: 149-159, 1987.

18. Priestley JV, Michael-Titus AT and Tetzlaff W: Limiting spinal cord injury by pharmacological intervention. Handb Clin Neurol 109: 463-484, 2012.

19. Basso DM, Beattie MS and Bresnahan JC: A sensitive and reliable locomotor rating scale for open field testing in rats. J Neurotrauma 12: 1-21, 1995. 
20. Stampas A and Tansey KE: Spinal cord injury medicine and rehabilitation. Semin Neurol 34: 524-533, 2014.

21. Quinzaños-Fresnedo J and Sahagun-Olmos RC: Micro RNA and its role in the pathophysiology of spinal cord injury-a further step towards neuroregenerative medicine. Cir Cir 83: 442-447, 2015 (In Spanish).

22. Haller J, Bice M and Lawrence B: Mediating the secondary effects of spinal cord injury through optimization of key physiologic parameters. J Am Acad Orthop Surg 24: 160-171, 2016.

23. Cai W and Shen WD: Anti-apoptotic mechanisms of acupuncture in neurological diseases: A review. Am J Chin Med 46: 515-535, 2018.

24. Oyinbo CA: Secondary injury mechanisms in traumatic spinal cord injury: A nugget of this multiply cascade. Acta Neurobiol Exp (Wars) 71: 281-299, 2011.

25. Mattson MP: Apoptosis in neurodegenerative disorders. Nat Rey Mol Cell Biol 1: 120-129, 2000.

26. Sunshine JE, Dagal A, Burns SP, Bransford RJ, Zhang F, Newman SF, Nair BG and Sharar SR: Methylprednisolone therapy in acute traumatic spinal cord injury: Analysis of a regional spinal cord model systems database. Anesth Analg 124: 1200-1205, 2017

27. Wilson JR, Jaja BNR, Kwon BK, Guest JD, Harrop JS, Aarabi B, Shaffrey CI, Badhiwala JH, Toups EG, Grossman RG and Fehlings MG: Natural history, predictors of outcome, and effects of treatment in thoracic spinal cord injury: A Multi-center cohort study from the North American Clinical Trials Network. J Neurotrauma 35: 2554-2560, 2018.

28. Karsy M and Hawryluk G: Pharmacologic management of acute spinal cord injury. Neurosurg Clin N Am 28: 49-62, 2017.

29. Zhang T, Li S, Li J, Yin F, Hua Y, Wang Z, Lin B, Wang H, Zou D, Zhou Z, et al: Natural product pectolinarigenin inhibits osteosarcoma growth and metastasis via SHP-1-mediated STAT3 signaling inhibition. Cell Death Dis 7: e2421, 2016.

30. Zhang T, Li S, Li J, Yin F, Hua Y, Wang Z, Lin B, Wang H, Zou D, Zhou Z, et al: Correction to: Natural product pectolinarigenin inhibits osteosarcoma growth and metastasis via SHP-1-mediated STAT3 signaling inhibition. Cell Death Dis 9: 902, 2018.
31. Lee HJ, Venkatarame Gowda Saralamma V, Kim SM, Ha SE, Raha S, Lee WS, Kim EH, Lee SJ, Heo JD and Kim GS: Pectolinarigenin induced cell cycle arrest, autophagy, and apoptosis in gastric cancer cell via PI3K/AKT/mTOR signaling pathway. Nutrients 10: pii: E1043, 2018

32. Wang C, Cheng Y, Liu H, Xu Y, Peng H, Lang J, Liao J, Liu H, Liu $\mathrm{H}$ and Fan J: Pectolinarigenin suppresses the tumor growth in nasopharyngeal carcinoma. Cell Physiol Biochem 39: 1795-803, 2016.

33. Csupor D, Widowitz U, Blazsó G, Laczkó-Zöld E, Tatsimo JS Balogh A, Boros K, Dankó B, Bauer R and Hohmann J: Anti-inflammatory activities of eleven Centaurea species occurring in the Carpathian Basin. Phytother Res 27: 540-544, 2013.

34. Gal P, Kravcuková P, Mokry M and Kluchová D: Chemokines as possible targets in modulation of the secondary damage after acute spinal cord injury: A review. Cell Mol Neurobiol 29: 1025-1035, 2009.

35. Cristante AF, Barros Filho TE, Marcon RM, Letaif OB and Rocha ID: Therapeutic approaches for spinal cord injury. Clinics 67: 1219-1224, 2012.

36. Song Y, Xue H, Liu TT, Liu JM and Chen D: Rapamycin plays a neuroprotective effect after spinal cord injury via anti-inflammatory effects. J Biochem Mol Toxicol 29: 29-34, 2015.

37. Wang YT,Lu XM, Chen KT, Shu YH and Qiu CH: Immunotherapy strategies for spinal cord injury. Curr Pharm Biotechnol 16: 492-505, 2015.

This work is licensed under a Creative Commons Attribution-NonCommercial-NoDerivatives 4.0 International (CC BY-NC-ND 4.0) License. 